\title{
Los prólogos y las dedicatorias en los textos traducidos de los siglos XIV y XV: Una fuente de información sobre la tra- ducción y la reflexión traductológica
}

\section{Rocío del RÍO FERNÁNDEZ}

\section{Introducción}

El presente artículo tiene por objeto abordar el estudio de los prólogos y las dedicatorias de algunas de las obras traducidas durante los siglos XIV y XV con el fin de demostrar, basándome en la información proporcionada en los mismos:

1) Que en el siglo XIV comienza a desarrollarse, aunque de manera todavía muy incipiente, la reflexión y la crítica sobre la traducción, que continuará evolucionando a lo largo del siglo XV hasta lograr su pleno auge en el siglo XVIII, y

2) Que la actividad traductora, llevada a cabo en ese mismo período (siglos XIV y XV), de textos latinos, en su mayoría, a las distintas lenguas romances existentes, contribuye a verificar la consolidación de la traducción en todas esas lenguas como instrumento cotidiano de transmisión de la cultura.

La razón que ha motivado la redacción de este trabajo ha sido la escasa información que, en mi opinión, existe al respecto. El punto de partida lo constituyen dos afirmaciones, realizadas por Julio César Santoyo en su libro Historia de la traducción: Quince apuntes, hace tan sólo cinco años, en 1999, que se pueden englobar en los dos objetivos que pretende este trabajo. La primera dice así: "Fue [en el siglo XIV] cuando se inició en España la reflexión [traductológica], si bien de forma tan tímida y escueta que apenas si satisface las expectativas del estudioso. [...] Y es que sólo en la segunda mitad del siglo XIV comienza a analizarse de modo embrionario la operación traductora, o sus instrumentos, o las condiciones en que se desarrolla; o al menos, sólo a partir de ese momento contamos con breves testimonios de tal análisis y reflexión (Santoyo 1999 a: 45-6). Además, esta situación no se da únicamente en la Península Ibérica, sino que se extiende al resto de Europa.

Ya en 1987, Santoyo había indicado en la introducción a su obra Teoría y crítica de la traducción: Antología, lo siguiente: El período de reflexión [traductológica] iniciado por Cicerón [año 46 a.C.] no da comienzo en España hasta la segunda mitad del siglo XIV (Santoyo 1987: 10). Y esa primera reflexión traductológica a mediados del siglo XIV surge [...] como una consecuencia más del espíritu renacentista, 
o prerrenacentista al menos, que comenzaba a alentar en la península; y va creciendo poco a poco y desarrollándose hasta alcanzar completa madurez en el siglo XVIII (Santoyo 1987: 12-3). Y añade: No obstante, estos comentarios [...], aunque breves en general, reflejan ya, anuncian e intuyen (a veces con sorprendente acierto) toda la variada problemática del traducir (Santoyo 1987: 13).

La segunda aseveración dice como sigue: La traducción [en el siglo XIV] se consolida en todas las lenguas romances [...] como vehículo habitual de difusión cultural (Santoyo 1999 a: 47) debido a su descentralización de la corte y del patronazgo real y a su dispersión por toda la geografía peninsular; puesto que durante los dos siglos anteriores, el XII y el XIII, había sido la monarquía, prácticamente en exclusiva, la que había "acaparado" y "monopolizado" la tarea de promover e impulsar la traducción, mediante la demanda y solicitud de versiones del árabe al latín y de éste al castellano, con el fin de enriquecer su propia cultura y de facilitar, a su vez, el acceso a la misma a todos aquellos lectores que desconocían la lengua original.

\section{Evolución del prólogo desde la Antigüedad clásica hasta la Edad Media}

En primer lugar, remontémonos brevemente al origen del prólogo, que tuvo lugar en Grecia y Roma. El prólogo en la Grecia clásica está relacionado con la introducción o inicio de una acción, la acción teatral de la tragedia. Esta acción teatral está constituida por al menos tres momentos que siguen un orden establecido: comienzo, medio y fin. Aristóteles (384-322 a.C.) considera el prólogo como una parte completa de la tragedia que "precede a la entrada del coro" (Montoya y Riquer 1999: 35), refiriéndose al instante en que el actor principal y su confidente ponían al público en situación relatándole los antecedentes de la obra y la tensión dramática que la había desencadenado, para dar paso al desarrollo de la acción teatral propiamente dicha.

Por lo que se refiere a la literatura latina, Plauto (ca. 254-184 a. C.) fue el primero que, en sus comedias, utilizó prólogos. En ellos expone los modelos griegos en los que se ha inspirado y la condición de su público. En el arte de la oratoria romana, que consta de cinco partes: inventio, dispositio, elocutio, memoria y pronunciatio, el prólogo toma el nombre de exordio y constituye el comienzo del discurso retórico o persuasivo dentro de la primera parte, la inventio. No obstante, hay discrepancias en torno a esa denominación. El célebre rétor Quintiliano (42-120 p.C.) se decanta en el capítulo 1 del libro IV de su tratado De institutione oratoria por la voz griega prooimion ('proemio') 
frente a la palabra latina exordium ('inicio') pues, en su opinión, aquella (prooimion) refleja de una manera más clara la localización y la función de esta parte en el discurso persuasivo: pro ('delante') y óimos ('camino'), es decir, lo que va delante de la obra abriendo el camino antes de adentrarse en el argumento de la misma.

Asimismo, Aristóteles establece en el capítulo 14 del libro III de su Retórica una diferenciación entre proemio, prólogo y preludio en función del género de la obra a la que éste preceda: prosa, poesía o pieza musical respectivamente: "El proemio está, pues, en el comienzo del discurso, lo mismo que el prólogo en la poesía y el preludio en la música de flauta. Todos ellos son comienzos, y como un desbrozar el camino para el recorrido de la oración (Mortara Garavelli 1991: 70)

En definitiva, todas estas definiciones contribuyen a conformar la acepción general de prólogo (del griego protos 'primero' y logos 'tratado') como aquello que antecede a la obra en sí, independientemente del tipo que sea (literaria, musical, etc.) y que es la que ha llegado hasta nuestros días ${ }^{1}$.

La Edad Media retoma y reelabora los modelos retóricos creados por los griegos y trasladados a los romanos, adecuándolos a las necesidades de la época. En el período medieval el propósito del prólogo estaba encaminado a atraer la atención de los lectores y disponerlos para que aceptasen la obra con benevolencia, según la tradición retórica heredada de Roma.

En su Rhetoric, hermeneutics, and translation in the Middle Ages. Academic traditions and vernacular texts del 1995 Rita Copeland dice: In Latin antiquity, it was the framework of rhetoric and grammar, and of the relationship between these two disciplines, that gave meaning to ideas about translation. For the Middle Ages as well, it was through the tradition of academic discourse, inherited from the Romans, that ideas about translation took shape (Copeland 1995: 9).

En las escuelas tanto griegas como romanas existían dos disciplinas relacionadas entre sí: la gramática, que comprendía el estudio de la lengua y el comentario textual de la misma, y la retórica, que consistía en la producción de discursos persuasivos. En Roma, la traducción tenía lugar en ambas disciplinas: In grammatical study it was an exercise; in rhetorical study it was considered both an exercise and an art form (Copeland 1995: 9). Según Copeland, la reflexión traductológica presente en las obras de Cicerón (106-43 a.C.) y Quintiliano

${ }^{1}$ El Diccionario de la Real Academia Española recoge la siguiente definición de prólogo en su primera acepción: Escrito antepuesto al cuerpo de la obra en un libro de cualquier clase. 
vanía a definir la condición de la retórica con respecto a la gramática: The theories of translation contained in the writings of Cicero and Quintilian were formulated, not with the express aim of defining the practice of translation itself, but rather as a way of defining the status of rhetoric in relation to grammar (Copeland 1995: 9).

Debido a la mala fama que tenía la profesión de traductor en esta época y a lo criticada que era, pues el descuido y la negligencia de algunos dejaba mucho que desear, los propios traductores admiten en esos prólogos y dedicatorias carecer de la preparación suficiente para traducir correctamente. Ahora bien, hemos de recordar que éste era, a su vez, un tópico -la captatio benevolentiae- muy utilizado por ellos a la hora de dirigirse por primera vez a sus lectores, con el fin de atraer su atención y su buena voluntad. Así pues, debemos desconfiar de esta fingida pose de excesiva modestia y de incapacidad para traducir.

Hay que tener en cuenta desde el primer momento que las opiniones de los traductores son útiles en cuanto que esclarecen muchos aspectos de la traducción, aunque no todos, pero también hay que tener presente, a efectos de estudio y de credibilidad de los mismos, la obligada dosis de retórica que cada prólogo y cada dedicatoria debía contener.

\section{Partes del prólogo}

El prólogo que precedía a las traducciones en la Edad Media proporcionaba todo tipo de información sobre quién había encargado la traducción, quién la había realizado, cuándo, por qué se había traducido esa obra y no otra y qué aportaba ésta a la cultura de llegada. El prólogo constaba de varias partes que carecían de un orden establecido. Éste solía ir precedido de una dedicatoria del traductor dirigida al mecenas que le había encargado la traducción, ensalzando su persona y su labor. A continuación, el traductor realizaba una reflexión sobre la traducción. Las reflexiones sobre la traducción durante la Edad Media aparecían sobre todo en los prólogos y epílogos.

A la hora de elaborar un prólogo, existían una serie de lugares comunes o tópicos, procedentes de la organización en partes del discurso retórico romano, que fueron recopiladas por Quintiliano en su De institutione oratoria. Estos tópicos fueron muy utilizados en la época medieval tanto en los prólogos literarios como en los prólogos a las traducciones, con el fin de disponer favorablemente a los lectores para la lectura de las obras. Entre ellos se encuentran cuatro fundamentales: 1. El tópico de la afectación de modestia, relacionado con el de la captatio benevolentiae, esto es, la confesión por parte del autor 
de su impericia para traducir, con el fin de atraer la buena voluntad e indulgencia de su público ante sus posibles errores en la traducción. Este recurso retórico era especialmente eficaz desde el punto de vista psicológico, puesto que existe una relación natural de empatía por parte del lector con aquel que se encuentra en dificultades. Podía utilizarse al principio, en el medio o al final del texto, siendo éste último lugar el más adecuado, pues los sentimientos estaban a flor de piel, una vez leído todo el prólogo; 2. La referencia mediante citas o alusiones al modo de traducir y a las ideas sobre el oficio de la traducción de los autores antiguos y clásicos; 3 . La explicación de la razón que induce al traductor a verter esa obra a otra lengua, que incluye los siguientes tópicos: la dedicatoria, la alabanza de la persona que ha encargado la traducción de la obra, el fingir no aceptar de muy buen grado el encargo de la traducción, la exposición de sus propios méritos y carencias; y la invocación a la divinidad y, por último, 4. La brevitas, expresada mediante los tópicos ex pluribus pauca o pauca e multis ('pocas, de entre las muchas cosas que podrían decirse'), menos empleado en los prólogos a las traducciones.

El prólogo fue utilizado sobre todo durante los siglos XIV al XVII, período en que alcanzó su mayor desarrollo, bajo distintos nombres: prefacio, proemio e introducción, entre otros. Esta situación fue propiciada por varias circunstancias: 1) la preponderancia de la palabra escrita sobre la oral, 2) la invención de la imprenta y el avance en las técnicas de reproducción del texto y 3) la importancia que fueron adquiriendo los mecenas como patrocinadores de la cultura.

3. Los prólogos de los textos traducidos en Europa y su importancia para el estudio de la reflexión traductológica

A continuación voy a exponer la relevancia que tienen los prólogos para el campo de la traducción, basándome en el ejemplo de prólogos concretos, además de ofrecer las muestras del comienzo y evolución de la reflexión traductológica en Europa que se pueden encontrar en los mismos, mediante el análisis de algunos de ellos. Dichos prólogos forman parte de traducciones realizadas en Francia, Italia y España.

\subsection{Los prólogos en las traducciones francesas}

Dice Christian Balliu, En Europa occidental habrá que esperar la Edad Media, y más exactamente en Francia el siglo XIV, para encontrar [...] huellas de reflexión traductológica, impresas en el limo de los prefacios (Balliu 1995: 41). El origen de estos prefacios, tan 
utilizados en las obras francesas durante el medievo, está relacionado con el impulso que recibió la traducción en esta época y la intensa labor realizada por dos reyes: Juan II el Bueno (1319-1364) y su sucesor en el trono Carlos V el Sabio (1337-1380). Su interés por la traducción los llevó a rodearse de traductores-consejeros como Pierre Bersuire, en el caso de Juan el Bueno, y a ser conscientes desde el primer momento de la necesidad de una estrecha colaboración entre el traductor y el experto en el tema, como le ocurrió al monarca Carlos V.

En el siglo XIV, por tanto, podemos hallar signos de reflexión traductológica en los prefacios de las traducciones francesas. La aparición de éstos fue debida a la existencia de numerosos decretos reales muy estrictos que obligaban a los traductores a justificar su trabajo en esos prólogos. Estas primeras palabras que anteceden a la traducción son en realidad el caldo de cultivo de los tratados sobre traducción posteriores.

Su finalidad es fundamentalmente didáctica y por esta razón el texto origen es susceptible de mejora, modificación y adaptación a la época contemporánea, según el pensamiento francés. Los prefacios [...] se caracterizan por una vertiente decididamente didáctica [...] y una apropiación del texto fuente (Balliu 1995: 41). Esta misma idea había sido recogida ya por Jacques Monfrin cuarenta años antes, en 1964: Suivant une idée généralement répandue, tout écrit destiné à instruire est perfectible et du moment qu'on le transcrit et qu'on le traduit, on ne voit aucune raison pour ne pas le modifier au goût du jour ou l'améliorer en le complétant à l'aide de renseignements puisés à d'autres sources (Monfrin 1964: 217-8).

En el siglo XV comienzan a elaborarse los prohesmes o prefacios de tipo argumentativo; los traductores realizan traduccionescomentario y se permiten hacer correcciones al autor del texto original. Hacia finales de este siglo asistimos al rejuvenecimiento de textos antiguos.

Por otra parte, los traductores medievales siguen insistiendo hasta el Renacimiento en demostrar la capacidad del romance francés como lengua de llegada para la traducción, en sustitución del latín, que aún era el idioma empleado en las Universidades, entre el clero, en la ciencia y en los documentos oficiales. Las palabras de Nicole Oresme (ca. 1320-1382), traductor de Aristóteles al vulgar francés, prueban la necesidad de la traducción a esta lengua: Sont pluseurs gens de langue françoise qui sont de grant entendement et de excelleent enging et qui n'entendent pas souffisanment latin, et pour ce les 
vaillans roys de France ont fait aucuns livres translater en françois (Monfrin 1964: 229).

Los 'prohesmes' franceses ponen en relación al traductor con el autor de la obra y asisten a la lengua meta, que tiene como foco de atención al lector. Asimismo, tratan acerca de los problemas de traducción derivados del carácter sintético de la lengua latina, en la que les constructions [étaient] si trenchies et si brieves, si suspensives et de si estranges mos, que diferían enormemente de la estructura del francés vernáculo, a la vez que ponían de manifiesto su escasez léxica frente a la riqueza del latín. De este modo, los traductores se veían obligados a emplear perífrasis y ampliaciones sintácticas en función del siguiente esquema: Ce qui semble trop brief je le allongeray, en exposant par mots et par sentences (Balliu 1995: 42).

Ya desde las primeras versiones de los clásicos al francés algunos traductores advierten que la raíz del problema que plantea el hecho de traducir no es solamente de carácter léxico, sino que también influye la estructura del francés que difiere totalmente de la del latín. Juan de Antioquia ya lo advirtió hacia el año 1282 en el epílogo a su traducción titulada Rettorique de Marc Tulles Cyceron: Ne les proprietez des paroles ne les raisons d'ordener les araisonemez et les diz dou latin ne sont pas semblables a celes dou françois (Russell 1985: 14). Y más tarde el traductor Raoul de Presles, que trasladó al francés la obra De civitate Dei de san Agustín, indica en su prólogo, dedicado al rey Carlos V el Sabio, que se distancia del texto original en favor de la veracidad, la simplicidad y la claridad: Et si je ne ensuis en ceste translacion les propres motz du texte et que je y voyse aucunes foys par une manière de circonlocution ou aultrement, il me sera pardonné pour ce que vous m'avez commandé, pour la matière esclarcir, que je ensuyve la vraye, simple et clere sentence et le vray entendement sans ensuyvir proprement les motz du texte (Balliu 1995: 42).

En esta cita queda patente la resistencia a traducir palabra por palabra, según el precepto de ese monarca, decisión que se mantendrá hasta la llegada del Renacimiento.

Así pues, el interés por verter obras en Francia se llevó a cabo con dos propósitos: 1. didáctico, es decir, poner la cultura al alcance de todos, y 2. lingüístico, esto es, establecer y afianzar los fundamentos de una lengua francesa que fuese capaz de rivalizar con la latina.

El traductor, no el autor, es el que garantiza la verdad científica y el que está capacitado, prácticamente con carácter divino, para revisar y enmendar su obra. La instrucción y la claridad en la forma de expresión priman a la hora de facilitar el acceso general a la cultura me- 
diante la traducción. Un dato curioso es el hecho de que la figura del traductor, presente a lo largo de toda la obra, aparece en miniaturas postrado a los pies del rey y mostrándole su traducción.

A lo largo del Renacimiento, los prólogos continúan precediendo a las obras, como en el caso de Claude de Seyssel (ca. 1450-1520), traductor del monarca Luis XII (1498-1515), que vertió al francés la Anábasis de Jenofonte y la Guerra del Peloponeso de Tucidides a partir de las versiones latinas intermedias que previamente había elaborado su ayudante Jehan Lascary, debido a su desconocimiento del griego. No obstante, los prólogos son cada vez menos abundantes a causa de la paralización que afecta a la traducción en Francia hasta el año 1530. A su vez, en el reinado de Francisco I (1515-1547) aumenta el número de traducciones, sin embargo, el de prefacios desciende significativamente por considerarse ya obsoletos.

De este hecho se deduce que la producción traductográfica, viva desde los tiempos más remotos, no se fundamentó en una visión traductológica organizada. Es más, no se fijan antes del siglo XVII los cimientos de una teorización de la actividad traductora; sólo aparecen reflexiones fugaces de traductores aislados que consignan observaciones acerca de su trabajo (Balliu 1995: 42).

La primera obra que puede ser considerada como el primer tratado de traducción en Francia fue elaborada por Gaspard de Tende (1618-1697) y editada en París en 1660, bajo el título Règles de la traduction, ou moyens pour apprendre à traduire de latin en françois, Tirez de quelques-unes des meilleures traductions du temps, también conocida posteriormente con el nombre de Tratado de traducción ${ }^{2}$. De Tende, además de contemplar el fin didáctico perseguido por los traductores jansenistas ${ }^{3}$, intenta aplicar a la traducción un alcance científico, que éstos habían obviado y que ya estaba presente en otras disciplinas como la gramática, a la vez que establece las reglas teóricas de la traducción y del perfecto traductor, extraídas a partir de la observación y el cuidadoso análisis de un buen número de textos traducidos. El prefacio de la obra lo recoge con estas palabras: Cela pourra estre utile, non seulement aux enfants \& à ceux qui les instruisent, mais encore à tous ceux qui veulent apprendre le Latin, puisque la Traduction

${ }^{2}$ A este tratado Christian Balliu dedica dos páginas en su artículo "Los traductores transparentes. Historia de la traducción en Francia durante el período clásico" en Hieronymus Complutensis, 1, 43-6.

${ }^{3}$ El Jansenismo es una doctrina religiosa del siglo XVII, creada por el teólogo holandés Jansen, que afirma que el hombre sólo puede alcanzar la salvación con la gracia divina. 
est sans doute un des mohines le plus court \& le plus facile pour apprendre les Langues (Balliu 1995: 43).

\subsection{Los prólogos en las traducciones italianas}

Italia constituyó desde sus orígenes una unidad cultural y geográfica rigurosamente delimitada y fue, además, el único país donde la tradición clásica pervivió como un elemento unitario a lo largo de los primeros siglos medievales. El latín, si bien cada vez menos puro, continuó siendo durante todo este tiempo la lengua popular y debido a esto no fue necesario, como en Francia o en España, la aparición temprana de una lengua romance que permitiera la comunicación con el pueblo. Pese a que cada región desarrolló dialectos propios a partir del latín, la lengua italiana no fue producto de un pueblo en busca de su identidad, sino la creación de un estamento intelectual que comprendió la necesidad de abandonar un latín cada vez menos flexible, y de proporcionar a la lengua vulgar la riqueza gramatical que le permitiera asimilar las nuevas tendencias.

En Italia la llegada del Humanismo, que se desarrolló durante los siglos XV y XVI, marca una oposición entre la manera de traducir antes y después de este movimiento. Las traducciones anteriores se caracterizan por ser eminentemente literales, debido probablemente al espíritu escolástico que predominaba en aquella época. Por si fuera poco, los traductores trabajaban con ejemplares manuscritos, con lo que en las traducciones se encontraban multitud de incoherencias.

Los traductores humanistas, cuyo más ilustre representante es Leonardo Bruni Aretino (1369-1444), discrepan de este modo de traducir y toman de nuevo los manuscritos griegos para volverlos a traducir al estilo renacentista, independientemente de las versiones escolásticas que ya circulaban. La traducción humanista defiende una cierta libertad estilística que proporcione a la traducción un estilo algo más elevado que la simple traducción literal.

De este modo, Bruni traduce directamente del griego al latín la Etica Nicomachea aristotélica -primera traducción renacentista de la obra del estagirita-, los discursos de Esquines y Demóstenes, y el tratado de san Basilio De legendis librorum gentilium, y explica concretamente en el prólogo a la Etica de Aristóteles, escrito en el año 1418, que la traducción de dicha obra se debió al "escándalo personal" que le provocó la versión realizada anteriormente por Robert Grossetête a la que tilda inflexiblemente de pueril, desmañada, áspera, de vocabulario embrollado, de absoluta y tosca rudeza, confusa e inadecuada en estilo, llena de errores y, en suma, hecha por un bárbaro deficiente 
en griego, deficiente en latín y en ninguna de las dos lenguas competente, acusándole de haber mancillado y adulterado el original aristotélico con esa traducción latina (Santoyo 1999 b: 32).

Por otro lado, dentro del Renacimiento existen también divergencias entre los propios traductores. Buena prueba de esto es la disputa que tuvo lugar hacia 1433, a raíz del mencionado prólogo, entre Leonardo Bruni y el obispo burgalés Alonso de Cartagena (13861456) -en la que también tomaron parte Pier Candido Decembrio y Poggio Bracciolini-, quien no compartía las ideas sobre la traducción expresadas por Bruni en su ensayo De recta interpretatione ${ }^{4}$, elaborado en 1425 ante las censuras que suscitó la severidad del prólogo a la Etica aristotélica, y en el que el humanista florentino afirma categóricamente ya desde el comienzo que toda la esencia de la traducción consiste en trasladar correctamente a una lengua lo que se ha escrito en otra, algo extremadamente difícil que no puede hacer quien no posea un gran dominio de ambas lenguas (Santoyo 1999 b: 32), la lengua de la que se traduce o lengua origen, en este caso el griego, y la lengua a la que se traduce o lengua meta, en este caso el latín. Y, lo que es más, el conocimiento de la lengua origen por parte del traductor no debe ser escaso ni vulgar sino más bien grande, suelto, esmerado, rico y estable, versado en la lectura de los filósofos, oradores, poetas y de todos los demás escritores (Vega 1994: 95).

Por otra parte, el traductor debe conocer y dominar la lengua meta, a fin de que cuando vaya a traducir una palabra por otra, no tenga que mendigarla ni la tome en préstamo ni la deje en griego por su ignorancia del latín, sino que conozca con precisión el valor y el sentido de las palabras (Vega 1994: 97). Asimismo, deberá tener muy en cuenta el uso y las figuras de dicción de los grandes escritores e intentará reproducirlos en sus traducciones y, sobre todo, evitará ser innovador en palabras y frases especialmente si no es apropiado. Para Bruni las características del buen traductor son: conocimiento de la materia y belleza de estilo.

De esta forma, el mejor método para traducir según Bruni es el siguiente: conservar de la mejor manera la estructura de la frase original, sin que las palabras traicionen el sentido ni el esplendor ni la

${ }^{4}$ Ver el artículo de Maurilio Pérez González. 1995. "Leonardo Bruni y su tratado De interpretatione recta" en Cuadernos de Filología Clásica: Estudios latinos, 8, pp. 193-233, mencionado en la bibliografía. Y ver asimismo la versión de Antonio Guzmán "L. Bruni. La traducción correcta" en Miguel Ángel Vega (ed.). 1994. Textos clásicos de teoría de la traducción. Madrid: Cátedra, 94-104, citado también en la bibliografía. 
belleza de las propias palabras (Vega 1994: 98), adaptándose al modo de expresión personal del autor original. En definitiva, y en palabras del propio Bruni, gran asunto, pues, y difícil es traducir correctamente (Vega 1994: 95).

En opinión del florentino, tres son los defectos del traductor: 1) la deficiente comprensión de lo que se debe traducir, bien sea por ignorancia del idioma o de la materia, 2) verterlo de manera contraria a como se debe en la lengua a la que se traduce, y 3) en lo que respecta a rasgos formales, traducir de tal modo que aquello que el autor original dijo apropiada y armónicamente resulte inadecuado, inarmónico y disforme (Santoyo 1999 b: 33).

Alonso de Cartagena tuvo conocimiento del prólogo a la Ética a Nicómaco de Aristóteles en Salamanca y se dispuso a responder, no con ánimo de molestar a Bruni, sino con la intención de interceder por el anterior traductor, del que destaca la utilización de grecismos ${ }^{5}$ que él considera apropiados, mientras que Bruni los tacha de vanos y superfluos, pues aunque existen errores, su traductor difundió la obra de buena fe y de la mejor manera que sabía, y por tanto no merece represión alguna sino elogios (Vega 1994: 94). Por su parte, Bruni reconoce su quizá excesivo enfado, debido a la irritación que le produce ver en qué lamentable estado había quedado en la versión latina de este traductor el contenido de los libros originales griegos que rezumaban elegancia, dulzura y un atractivo incalculable; y se justifica haciendo alusión a la reacción de traductores de la talla de san Jerónimo (342-420) y Marco Tulio Cicerón en situaciones similares ${ }^{6}$.

Sin embargo esa defensa de Alonso de Cartagena no se tiene en pie por una razón: Cartagena desconocía absolutamente el griego, y así lo admite abiertamente; es decir, carecía de los conocimientos necesarios para juzgar eficazmente la traducción. De este modo, su examen se basaba únicamente en la calidad del texto latino, obviando cualquier alusión al texto original y provocando la risa en su oponente, quien recibió esta respuesta tres años más tarde, en 1436, por medio del arzobispo de Milán, Pizzolpasso. Un año después, en 1437, Alonso de Cartagena envió otra carta a Leonardo Bruni, a través nuevamente del arzobispo de Milán, en la que aquél continuaba la controversia y, en opinión del Aretino, su persistencia en el error. La respuesta del

${ }^{5}$ Se pueden ver ejemplos en Miguel Ángel Vega (ed.). 1994. Textos clásicos de teoría de la traducción. Madrid: Cátedra, 103-104.

${ }^{6}$ Ver Miguel Ángel Vega (ed.). 1994. Textos clásicos de teoría de la traducción. Madrid: Cátedra, 104. 
italiano no se hizo esperar y su contestación fue aún si cabe más convincente: No le corresponde al traductor adivinar, ni conjeturar, ni escribir lo que a él le parece, ni escorar las palabras hacia su propia opinión, sino simple y directamente, sin mutación alguna, tal como está en el otro idioma, así referirlo en el suyo; pues al traductor no le está permitido introducir variantes (Santoyo 1999 b: 35).

Una vez dejado claro el oficio del traductor en esta particular reflexión sobre la traducción, retoma la polémica comenzada por Cartagena y le responde de la siguiente manera: No veo, la verdad, cómo [Alonso de Cartagena] puede tener en esto opinión, pues cuando se habla de la fidelidad de una traducción es necesario apelar al significado de las palabras griegas; porque si traducir no es sino expresar una lengua en otra, como él no conoce ni entiende una de las dos, sólo puede discutir de esto por opiniones y adivinanzas (Santoyo 1999 $b$ : 35) de modo que, o está haciendo trabajo de adivinación, y no de investigación, o quiere polemizar sobre lo que Aristóteles debiera haber dicho, y no sobre lo que dijo (Camillo 1976: 212).

Es evidente que en esta polémica la balanza se inclinó del lado de Bruni, pero no por eso debemos despreciar la aportación y la incipiente reflexión traductológica llevada a cabo por Cartagena, quien, a diferencia de Bruni, no expuso sus ideas en una obra aparte sino en los prólogos a sus traducciones.

Cartagena postulaba que en los textos filosóficos era más fiable una traducción ad sensum ('según el sentido'), siguiendo a san Jerónimo, y ajustada al contenido del texto original puesto que de este modo transmitía el pensamiento del autor de una manera más directa, exacta y veraz; por esta razón no le importaba que en la traducción existiesen muchas palabras griegas, más aún cuando éstas reproducían el sentido de una manera más clara y concisa y cuando los equivalentes latinos, que proponía Bruni, no eran adecuados para transferir el significado de Aristóteles. Cartagena, en definitiva, se aproximaba a los escritores clásicos de manera más imparcial y objetiva que Bruni, y supeditaba la retórica al contenido pues aquella impedía la correcta comprensión de éste. El prelado burgalés, en oposición a Bruni, se guiaba en sus traducciones por el siguiente dictado: es el espíritu de un texto, no la letra, lo que debe ser respetado (Camillo 1976: 219).

Bruni, por su parte, insistía en que el sentido de las palabras latinas empleadas en su traducción se basaba en Cicerón y Séneca: los mejores autores clásicos y más dignos de confianza, incluso utilizaba su estilo y dicción con objeto de recuperar la elocuencia aristotélica, 
pero Cartagena no consideraba esta fidelidad a los clásicos como garantía suficiente de una buena traducción.

No es que el obispo de Burgos se opusiese al lenguaje de los clásicos Marco Tulio Cicerón o Lucio Anneo Séneca (3 a.C.-65), sino a la utilización que Bruni hacía de él, puesto que, según nos informa Cartagena dado su amplio saber acerca del pensamiento moral antiguo, el conocimiento que ambos escritores latinos poseían de la Ética de Aristóteles era imperfecto o nulo, de ahí lo arriesgado de tomar palabras de estos autores para verter al latín conceptos aristotélicos. Por tanto, en lo que respecta a la filosofía moral, la terminología de Cicerón, como la de Séneca y otros estoicos, es inaplicable a la traducción de Aristóteles (Camillo 1976: 221), idea que Bruni no rebatió en su respuesta a la epístola de Cartagena, lo que indica su conformidad con la misma.

A diferencia de la mayoría de los humanistas italianos, que se decantaban por un latín clásico al que sólo podían acceder muy pocos lectores, Cartagena opta por un latín no necesariamente clásico sino también medieval o incluso palabras de uso cotidiano y utiliza la lengua vernácula, a la que traduce a Cicerón y a Séneca ${ }^{7}$ con el fin de llegar al mayor número de lectores, pues en su opinión el saber y las ideas solamente tienen valor si comunicados, y si aplicados al bienestar de la sociedad (Camillo 1976: 219), a pesar de su admiración por la elocuencia clásica y la de Bruni. La claridad, la sencillez y la precisión de Cartagena contrastan con la oscuridad, el purismo y la artificialidad de Bruni.

Si algún que otro crítico ha visto en esta polémica una disputa entre el escolasticismo y el humanismo, o entre la filología renacentista y la dialéctica medieval, otros han visto una intención de restaurar la unión entre el aristotelismo y el cristianismo. Sin embargo, basta una lectura de la correspondencia entre el resto de participantes en la disputa -el arzobispo de Milán Pizzolpasso, Pier Candido Decembrio y Poggio Bracciolini- para comprobar que no se trataba de un mero enfrentamiento entre el pensamiento medieval y el humanista, como había sucedido en tantas otras ocasiones, sino de una "controversia [que] tuvo lugar entre dos hombres cultos, que perseguían objetivos más o menos semejantes, aunque por distintos caminos (Camillo 1976: 217).

${ }^{7}$ Alonso de Cartagena tradujo De officiis, De senectute, Rethorica, De inventione y Oratio pro Marcello de Cicerón, y los tratados De vita beata, De providentia y Declamationes de Séneca. 
Por último, hay que dejar claro que el carácter de la segunda misiva de Leonardo Bruni a Cartagena es ya conciliador, hasta el punto de que años después éste escribirá al obispo burgalés recordándole amistosamente la polémica y solicitando nada menos que su opinión sobre una obra suya: Isagocicon moralis disciplinae (Introducción a la filosofía moral), que había remitido al rey Juan II de Castilla.

\subsection{Los prólogos en las traducciones de la Península Ibérica}

Por lo que respecta a la Península Ibérica, siguiendo a Peter Russell (1985: 5), es ya bien entrado el siglo XVI cuando los traductores aceptan finalmente la lengua vulgar como vehículo eficaz para la transmisión de los contenidos de las distintas obras que por aquel entonces eran objeto de traducción y ponerlas, de este modo, al alcance de los legos. Posiblemente esta circunstancia fue debida a que el castellano ya estaba bastante consolidado como lengua. Recuérdese que Antonio de Nebrija había publicado su Gramática de la lengua castellana en 1492, con la consecuente repercusión que este tratado gramatical, el primero escrito en romance, tuvo a todos los niveles en la Península Ibérica: cultural, social, político y especialmente linguístico.

Con anterioridad, en los siglos XIV y XV, la insuficiencia de recursos que poseían las lenguas romances impedía una adecuada traducción de los textos latinos al castellano, al catalán y al gallego. Los traductores, conscientes de esta situación, estimaron oportuno exponer al lector las dificultades que esta circunstancia les planteaba a la hora de traducir correctamente un texto. El lugar elegido para realizar sus quejas y observaciones al respecto fueron los prólogos y las dedicatorias que precedían a las traducciones, donde los traductores expresaban su opinión acerca del oficio de traducir y su reflexión sobre el concepto de traducción. Por vez primera se empieza a considerar la enorme complicación que supone la tarea de verter el texto de una a otra lengua.

Como indica Henry Van Hoof acerca de la reflexión sobre la traducción: Des considérations sur la traduction il y en eu certes au cours des siècles [...], mais c'était toujours sous forme de préfaces aux oeuvres traduites, de manuels a visée didactique [...] ou des pasajes occasionnels dans des ouvrages plus généraux (1998: 21).

No obstante, llama la atención el hecho de que, a la vez que se producen este tipo de quejas por parte de los traductores acerca de la problemática que plantea la actividad de traducir, el volumen de textos traducidos no deja de aumentar en ambas Coronas, la de Castilla y la de Aragón. 
Al contrario que en Francia, en la Península Ibérica, y más de un siglo después con respecto a ese país, el problema que plantea mayor dificultad para los traductores del latín al romance es el lexicográfico, según informan al lector en sus prólogos; es decir, por lo general, los comentarios de los traductores españoles no profundizan en la problemática de la traducción, sino que se limitan a recoger cuestiones de vocabulario. Los traductores muestran, en general, un considerable desconocimiento de las dificultades que implica la actividad de traducir en sí misma, apenas dedican alguna línea a la exposición de los problemas concretos que les plantea su tarea, los comentarios son más bien escasos y casi siempre se reducen a las fórmulas tradicionales sobre el tema, omitiendo con frecuencia su experiencia personal sobre los problemas que supone la traducción. Por tanto, la reflexión traductológica en esta época es aún muy superficial, pero, a pesar de todo, hay alguna destacada excepción como es el caso de Alonso Fernández de Madrigal, el Tostado, que, a modo de precursor y crítico, representa una amplia e importante aportación sin precedentes en la Península.

En cuanto a las consideraciones sobre la teoría de la traducción, siguen, por lo general, las normas tradicionales, que tenían sus fundamentos en las obras clásicas y de teología de los santos Padres, con el inconveniente de que estas normas habían sido creadas para un propósito diferente y estaban enmarcadas en otro ámbito lingüístico y cultural distinto al de trasladar un texto escrito en latín a una lengua romance, de modo que resultan obsoletas para las necesidades de esta época. Por tanto, la reflexión teórica no existía, y se caracterizaba por el interés práctico e inmediato de transferir de una lengua a otra y por la falta de método.

No obstante, el comentario de la introducción puede, en ocasiones, y siempre dentro de las normas que marca la tradición, ampliarse y desembocar en un razonamiento basado, cuando menos en parte, en alguna de las situaciones en que se haya visto el traductor. Así ocurre, por ejemplo, en el pròlech del traductor mallorquín Ferrán Valentí a su traducción del latín al catalán de los Paradoxa de Cicerón (ca. 1450): Yo Ferrando Valentí, inerudit e dexeble dels dexebles, he posada e transferida aquesta petita obreta de Tul.li, gran en sentencia de latí en vulgar materno e malorquí [...], alcunes paraules e a les voltes tolent de la textura literal de aquella, no però tocant en sentència alcuna, ans per retre aquella clara e perceptible, e alcuna volta transferint de mot per no mudar sentencia en aquella (Santoyo 1987: 42). 
También hace notar en dicho pròlech el tópico de la falsa modestia: Perquè ha paregut a tu alcunes de aquelles ambiguïtats ésser tractades en aquel petit libre per Tul-li ordenat e compost, lo cual se intitola Paradoxe, has a mi no solament pregat, mes turmentat $e$ forçat, volgués per causa tua e erudició aquelles dites Paradoxes de latí en vulgar transferir, creent tu yo ésser en tals coses suficient e abte, la qual cosa és molt luny de la veritat (Santoyo 1987: 42).

$\mathrm{Y}$ se hace responsable ante el lector de los errores cometidos en la traducción: E per ço tu, legidor, si per ventura veuràs e conexeràs les dites coses, pren-ho per aquesta intenció; yo a present te dich e.t manifest que totes errós, inèpcies e grosseries veuràs e legiràs en la dita obra; però no.t penses sien del actor de aquella, mes digues $e$ verdaderament cregues són de mi, indigne, ignorant e atrevit transladador (Santoyo 1987: 42).

Por tanto, algunos traductores reconocen de manera explícita que no han traducido bien, pero coinciden en señalar, como indicaba don Iñigo López de Mendoza, marqués de Santillana, en la carta escrita a su hijo, Pedro González de Mendoza, para que tradujera al romance la Ilíada de Homero, que siempre es mejor disponer de la traducción de una obra clásica, aunque sea imperfecta, que carecer totalmente de acceso a ella: Pues non podemos aver aquello que queremos, queramos aquello que podemos. É si caresçemos de las formas, seamos contentos de las materias (Santoyo 1987: 38)

\subsubsection{Corona de Aragón: Características de los prólogos a las traducciones \\ Gracias al interés de los príncipes de la Casa de Barcelona por la} cultura y a la óptima relación que éstos mantenían con la corte francesa, comenzaron a realizarse a partir del siglo XIV un buen número de traducciones al romance catalán, unas veces a partir de versiones francesas intermedias, y otras directamente del latín tanto clásico como medieval, con el propósito de acercar a los lectores las grandes obras clásicas latinas.

En la corona de Aragón, siguiendo la división establecida por Roxana Recio (1996: 143), se pueden distinguir tres grandes grupos de traductores de los autores clásicos latinos desde finales del siglo XIV y durante todo el XV.

El primero está constituido por aquellos que, a la manera de la corona de Castilla, se decantan por una traducción literal, muy pegada a la lengua de partida, esgrimiendo el argumento de la suptilitat del latín frente a la inferioridad de la lengua vernácula, es decir, los tra- 
ductores catalanes se lamentan de la imposibilidad de trasladar al romance la suptilitat de la lengua latina, término utilizado "para referirse a aquella especie de cualidad total del latín" (Russell 1985: 12). Se trata de traductores fundamentalmente latinistas. Seguidores de esta corriente son Jacme Conesa y Ferrer Sayol, entre otros. El traductor catalán Jacme Conesa se hace eco de esta situación en el prólogo a su traducción de la Historia destructionis Troiae, escrita originariamente por Guido delle Colonne a fines del siglo XIII, y llevada a cabo en 1367 a instancias del rey Pedro IV de Aragón del que era protonotario: Si algunes paraules seran transportades, o que paregua que no sien conformes de tot en tot al lati, no sia inputat a ultracuydament de mi, mas que cascu entena que aquel transportament o mudament es per donar antendre plenament e grosera los latins qui son soptils al dit noble hom et tots altres (Santoyo 1987: 23).

Asimismo, recurre al tópico de la afectación de modestia unas líneas más abajo: En cara mes pot esser imputat a (i)gno(ran)cia mia, qui segons la suptilitat de aquel qui les composa no so bastant ni suficient a fer tal traslatacio de lati en Romanz (Santoyo 1987: 23).

El otro traductor catalán, Ferrer Sayol, alude además, en su prólogo a la traducción de la obra De re rustica de Palladio efectuada en 1385, a otra característica del latín frente al catalán, la breuedat, y afirma haberse visto sorprendido por ella: La grant suptilidat, $e$ breuedat, $e$ vocablos que non son en uso entre nosotros en Cataluñya, nin aun en España (Santoyo 1987: 24).

Sayol lamenta la falta de preparación de los traductores de las anteriores versiones de esta obra reprochándoles su impericia y el exceso de latinismos que dificultan la comprensión del texto en romance: Algunos se sean fechos arromançadores, los quales non han auido cura de arromançar muchos vocablos, los quales non son conosçidos nin usados en nuestro lenguaje, mas han los puestos sinplement segunt que los han fallados escriptos en el Latin, en tanto que si poco son entendidos en el latin, asy tan poco son entendidos en el romançe (Santoyo 1987: 24).

Y lo que es aún más grave, la falta de fidelidad al texto original en muchas ocasiones: $E$ aun en muchas partidas del romançe non han expresado nin dicho el entendimiento de Palladio, antes han puesto el contrario en grant derogaçion e perjuyzio de Palladio (Santoyo 1987: 24).

Así pues, justifica su traducción explicando que no está conforme con ninguna de las que se han realizado hasta el momento, recurriendo al tópico de la falsa modestia: E yo, por todas aquestas cosas, he que- 
rido nueuamente arromançar e declarar, tanto quanto la mi groseria e insufiçiencia ha bastado, el dicho libro de Palladio, tornando aquel nueuamente de latin en romançe (Santoyo 1987: 24).

$\mathrm{Y}$ se disculpa por los errores que haya podido cometer, a pesar de su diligencia: Sy por auentura yo non he bien interpretados algunos vocablos [...] aquesto ha seydo porque non los he fallados expuestos nin declarados en algunos libros [...] ya sea que diligentemente en ello aya trabajado, e dexolo a correcçion de mayor e mejor interpretador que yo (Santoyo 1987: 24).

Un segundo grupo de traductores del latín al romance en la Corona de Aragón lo conforman aquellos que se permiten realizar un tipo de traducciones más libres. Así pues, amplían información con respecto al original y reducen o incluso suprimen la que no consideran relevante, e insertan fragmentos pertenecientes a otras obras que complementan y, sobre todo, clarifican el contenido de su versión. A este grupo pertenece el traductor Antoni Canals (ca. 1352-1419), que incluyó en su traducción del África de Petrarca párrafos completos de Tito Livio, con el fin de hacer la obra más comprensible al lector.

El tercer y último grupo se caracteriza por advertir, aunque sin haberlo comprobado en la práctica, el cúmulo de dificultades que supone la traducción literal. Su representante es Ferrán Valentí. Este traductor está a favor del transvase de textos latinos a la lengua vulgar: Alcunes doctrines estar amagades e secretes per ignorància de la lengua o idioma en lo qual éran stades posades per lo inventor o componedor de aquellas, la manifestació de les quals, si en lengua o parlar inte-ligible fossen tornades, fóra cosa molt aprofitable a molts (Santoyo 1987: 40).

Fue discípulo de Leonardo Bruni en Italia y a través de él aprendió a valorar la labor del traductor, a la que considera un trabajo moderno: Los moderns e moderníssims, posant e transferint molts libres $e$ obres de una lengua en altra, que per raó poguessen a molts valer $e$ aprofitar (Santoyo 1987: 40).

\subsubsection{Corona de Castilla: Características de los prólogos a las traducciones \\ Con relación a Francia e Italia la traducción de obras clásicas al romance se inicia en Castilla casi un siglo más tarde, sin embargo, una vez puesta en marcha, esta actividad traductora continúa con gran fuerza. A finales del siglo XIV, los vínculos políticos establecidos en- tre Castilla y Francia propician que las versiones francesas sirvan de modelo a los traductores castellanos para la elaboración de las suyas,}


como Pedro López de Ayala, primer traductor de Tito Livio al castellano, que se basó en la traducción de ese autor llevada a cabo por Pierre Bersuire para el monarca francés Juan el Bueno.

En el último decenio del siglo XIV y principios del XV se produce en Castilla una vuelta a las ideas de san Jerónimo. Esta circunstancia contrapone la tendencia tradicional que practica la traducción palabra por palabra, debido a la escasez de recursos de la lengua romance, a una tendencia más liberal, que sigue la teoría jeronimiana de traducir por el sentido.

Los comentarios de los traductores de Castilla hacen referencia a la dulçura del latín frente al romance castellano. Con este vocablo tal vez se quería expresar la "armonía acústica o tonal" y "las cadencias del latín", así como "las sutilezas ligüísticas y estilísticas" que mencionaban los traductores catalanes, y "la plasticidad y variedad de la expresión" que permitía esa lengua en oposición a las carencias del castellano (Russell 1985: 13). Así el traductor Pedro de Chinchilla señala en el prólogo a su traducción del latín al castellano de la Historia troyana de Guido delle Colonne -llevada a cabo en 1443 a petición del conde de Benavente, don Alfonso Pimentel- la falta de dulçura de la lengua castellana: Como ya otros la ayan al nuestro romançe tornado en asaz alto e dulçe estilo segunt la sufiçençia de nuestra lengua, en la qual si los conçebimientos mentales en la latina ystoria contenidos non han seydo tan conplidamente declarados ninguna culpa a ellos imputada ser deue, mas a la insufiçiente lengua en la qual el dulçe $e$ buen orden de fablar, segunt que en la latina, fallar non se puede (Schiff 1970: 267).

El marqués de Santillana, don Íñigo López de Mendoza, también reconoce que el castellano está desprovisto de la dulçura o graçiosidat propia del latín en la carta que dirige a su hijo Pedro González de Mendoza para pedirle que traduzca la Ilíada de Homero al castellano a partir de la versión latina en prosa de Pier Cándido Decembrio: Bien sé yo agora que, segunt ya otras veçes con vos é con otros me ha acaesçido, diredes que la mayor parte ó quassi toda de la dulçura ó graçiosidat quedan é retienen en sí las palabras é vocablos latinos (Santoyo 1987: 38).

Finalmente, otro aspecto que aparece mencionado insistentemente en los comentarios de los traductores no sólo castellanos sino también catalanes, como ya se ha visto, y peninsulares en general es la breuedat de la lengua latina. Pedro González de Mendoza menciona esta dificultad en el prólogo a la traducción en prosa castellana de la Ilíada de Homero (ca. 1442) a él atribuida: Los açentos, cuento e me- 
dida no se guarden commo en el uerso, porque muy grand parte de su fermosura pierde la dulce oración. [...]. De lo qual se sigue no la elocuençia como trompa resonante e arte famosa suya, mas algund tanto de las altas inuençiones e sentencias podamos conosçer (Schiff 1970: $5)$.

Esta circunstancia impide alcanzar, por consiguiente, la perfección expresiva del original en la lengua vulgar, pues: nos no auemos tan conpendiosos uocablos para que en pocas palabras pudiessemos conprehender grandes sentencias. Commo sea que la eloquençia de fuerças caresca quando el ydioma uocablos no padesçe diuersos respectos significantes (Schiff 1970: 5-6).

\section{La reflexión y la crítica traductológicas}

No tenemos noticia de ninguna reflexión sobre el arte de transvasar textos de una lengua a otra que se realizara en la cultura griega. La primera aportación teórica sobre la traducción nos llega, pues, de la civilización romana, y concretamente de la mano de Cicerón (106-43 a.C.). En su obrita De optimo genere oratorum, del año 46 a.C., que sirve de prólogo a la traducción de los conocidos discursos enfrentados de Esquines y Demóstenes -los dos oradores áticos más importantes-, analiza cómo llevó a cabo esta actividad (capítulos 13 y 14): No lo traduje como intérprete, sino como orador, con la misma presentación de las ideas y de las figuras, si bien adaptando las palabras a nuestras costumbres. En los cuales [discursos] no me fue preciso traducir palabra por palabra, sino que conservé el género entero de las palabras y la fuerza de las mismas. No consideré oportuno el dárselas al lector en su número, sino en su peso (Vega 1994: 77).

Más adelante el poeta latino Horacio (65-8 a.C.) recoge en su Epistola Ad Pisones, también conocida como Arte poética, los siguientes versos, que posteriormente cita san Jerónimo en su Epistola a Panmaquio: No trates de verter, escrupuloso / intérprete, palabra por palabra (Vega 1994: 85) La reflexión traductológica fue continuada por san Jerónimo (ca. 331-420), en la Carta a Panmaquio o De optimo genere interpretandi (ca. 400) donde justifica su manera de traducir (capítulo 5): Yo no solamente confieso, sino que proclamo en alta voz que, aparte las sagradas Escrituras, en que aun el orden de las palabras encierra misterio, en la traducción de los griegos no expreso palabra de palabra, sino sentido de sentido. Y tengo por maestro en esta parte a Tulio (Vega 1994: 84).

Posteriormente, san Agustín, Maimónides, el rey de Inglaterra Alfredo el Grande, Roger Bacon, Dante y Leonardo Bruni fueron, en- 
tre otros, los llamados a continuar la reflexión sobre la traducción a lo largo de la historia.

Por último, la reflexión y la crítica traductológicas propiamente dichas son dos fenómenos que comienzan a producirse de manera incipiente a finales de la Edad Media y principios del Renacimiento. Este período de reflexión y crítica continúa desarrollándose hasta lograr su completa plenitud en el siglo XVIII.

\section{Conclusión}

La segunda mitad del siglo XIV y el siglo XV constituyen un período en el que por vez primera se empieza a considerar la enorme complejidad que supone la tarea de verter una obra de una a otra lengua, como se ha podido comprobar a través de la información facilitada en los prólogos analizados.

Los prefacios franceses constan de una parte didáctica e instructiva por un lado y de adaptación del texto origen a la época y a la lengua vulgar que se traduce por otro, incluyendo modificaciones y mejoras con el fin de completar el texto original. Así pues, el objeto de los "prohesmes" consiste en analizar las dificultades que plantea la lengua latina para su traducción al francés, entre ellas: las características propias de cada lengua, que no se correspondían, y la riqueza léxica del latín frente al francés, incipiente en aquella época.

En los prólogos italianos se critica la versión medieval de los textos clásicos griegos al latín debido a su literalidad y tosquedad en favor de una traducción basada en la retórica y en el estilo de autores como Cicerón o Séneca, con el fin de rescatar, de este modo, la elocuencia griega. Aunque este método tuvo sus detractores, entre ellos Alonso de Cartagena, quien anteponía el contenido del texto, en materia de filosofía moral, a la retórica y defendía una traducción más cla$\mathrm{ra}$, sencilla y precisa que llegase a un mayor número de lectores.

Los traductores catalanes se muestran incapaces de reproducir la suptilitat de la lengua latina, mientras los castellanos indican que su lengua carece de la dulçura del latín y destacan la dificultad que plantea el vocabulario latino. Tanto éstos como los franceses insisten en la imposibilidad de igualar en su lengua la breuedat o carácter sintético de la latina; asimismo estos últimos señalan que la estructura del francés difiere totalmente de la del latín.

Quedan demostrados, pues, los dos objetivos pretendidos:

En primer lugar, que a través de los ejemplos de prólogos analizados, la fecha de la aparición de la reflexión traductológica en todos los países, bien sea Francia, Italia o la Península Ibérica, se sitúa en la 
segunda mitad del siglo XIV y continúa su evolución durante el siglo $\mathrm{XV}$. Esta circunstancia supone un avance con relación a la reflexión y la crítica traductológicas hasta el momento y deja constancia de la importancia de los prólogos y las dedicatorias como lugares físicos en los que se realiza esa reflexión.

$\mathrm{Y}$ en segundo lugar, que las traducciones a las distintas lenguas romances realizadas en esos dos siglos (XIV y XV) convierten a la traducción en un instrumento más de transmisión de la cultura y de consolidación, enriquecimiento y evolución de esas lenguas.

\section{Referencias bibliográficas}

BALLIU, Christian 1995, "Los traductores transparentes. Historia de la traducción en Francia durante el período clásico", en Hieronymus Complutensis 1, pp.9-51.

CAMILlO, Ottavio di 1976, El humanismo castellano del siglo XV, Valencia (Fernando Torres Editor).

COPELAND, Rita (1991) 1995, Rhetoric, hermeneutics, and translation in the Middle Ages. Academic traditions and vernacular texts, Cambridge (Cambridge University Press).

DOMÍNGUEZ BORDONA, Jesús (ed.) (1486) 1969, Fernando del Pulgar. Claros varones de Castilla, Madrid (Espasa-Calpe).

DOMÍNGUEZ BORDONA, Jesús (ed.). (1455) 1924, Fernán Pérez de Guzmán. Generaciones y semblanzas, Madrid (Ediciones de 'La Lectura').

FERNÁNDEZ VALLINA, Emiliano 1998, "Del Tostado sobre la traducción", en Maurilio Pérez González (coord.), Actas del Congreso Internacional sobre Humanismo y Renacimiento Vol. I, León (Universidad de León), pp.319-329.

LÓPEZ GARCÍA, Dámaso (ed.). 1996, Teorías de la traducción: Antología de textos, Cuenca (Universidad de Castilla-La Mancha).

MONFRIN, Jacques 1964, "Humanisme et traduction au MoyenÂge", en Actes du Colloque de l'Université de Strasbourg, París (Klincksieck).

MONTOYA MARTÍNEZ, Jesús y RIQUER, Isabel de (1998) 1999, El prólogo literario en la Edad Media, Madrid (UNED).

MORTARA GARAVELLI, Bice (1988) 1991, Manual de retórica, (trad. M ${ }^{\mathrm{a}}$ José Vega de Manuale di retorica), Madrid (Cátedra).

PAREDES, Juan y MUÑOZ RAYA, Eva (eds.). 1999, Traducir la Edad Media. La traducción de la literatura medieval románica, Granada (Universidad de Granada). 
PÉREZ GONZÁLEZ, Maurilio 1995. "Leonardo Bruni y su tratado De interpretatione recta", en Cuadernos de Filología Clásica. Estudios latinos 8, pp.193-233.

PÉREZ GONZÁLEZ, Maurilio 1996, "La traducción literaria según Leonardo Bruni", en Eustaquio Sánchez Salor \& al. (eds.) La recepción de las artes clásicas en el siglo XVI, Cáceres (Universidad de Extremadura), pp.377-384.

PÉREZ GONZÁLEZ, Maurilio 1999, G. Manetti y la traducción en el siglo XV. Edición crítica del Apologeticus, Libro V, León (Universidad de León).

RECIO, Roxana (ed.). 1995, La traducción en España ss. XIV-XVI [Anexos de Livius, 1], León (Universidad de León).

RECIO, Roxana 1996, "Por la orden que mejor suena': Traducción y Enrique de Villena", en La Corónica 24 / 2, pp.140-153.

RUSSELL, Peter 1985, Traducciones y traductores en la Península Ibérica (1400-1550). Bellaterra (Universidad Autónoma de Barcelona).

SANTOYO, Julio César 1987, Teoría y crítica de la traducción: Antología, Bellaterra (Universidad Autónoma de Barcelona).

SANTOYO, Julio César 1999a, "El siglo XIV: Traducciones y reflexiones sobre la traducción", en Historia de la traducción: Quince apuntes, León (Universidad de León), pp.35-50.

SANTOYO, Julio César 1999b, "La reflexión traductora en la Edad Media: Hitos y clásicos del ámbito románico", en Juan Paredes y Eva Muñoz Raya (eds.) Traducir la Edad Media. La traducción de la literatura medieval románica, Granada (Universidad de Granada), pp.21-42.

SCHIFF, Mario (1905) 1970, La bibliothèque du marquis de Santillane. Ámsterdam (Gérard Th. Van Heusden).

STEINER, George (1975) 1992, After Babel. Aspects of language and translation, Oxford (Oxford University Press).

VAN HOOF, Henry 1998, "Esquisse pour une histoire de la traduction en Espagne", en Hieronymus Complutensis 6-7, pp.9-23.

VEGA, Miguel Ángel (ed.). 1994, Textos clásicos de teoría de la traducción, Madrid (Cátedra). 